\title{
Spatial Restriction of Light Adaptation and Mutation-Induced Inactivation in Fly Photoreceptors
}

\author{
Baruch Minke and Richard Payne \\ Department of Neuroscience, The Howard Hughes Medical Institute, The Johns Hopkins University School of Medicine, \\ Baltimore, Maryland 21205
}

\begin{abstract}
The spatial spread within fly photoreceptors of 2 forms of desensitization by bright light have been investigated: the natural process of light adaptation in normal Musca photoreceptors and a receptor-potential inactivation in the nosteady-state (nss) mutant of the sheep blowfly Lucilia. The suction-electrode method used for recording from vertebrate rods was applied to fly ommatidia. A single ommatidium in vitro was partially sucked into a recording pipette. Illumination of the portion of the ommatidium within the pipette resulted in a flow of current having a wave form similar to that of the receptor potential and polarity consistent with current flow into the illuminated region of the photoreceptors. Two 5- $\mu \mathrm{m}$ slits of light, positioned at right angles to the ommatidial axis, were employed to determine the spread of light adaptation or inactivation along the ommatidium. The intensity of a flash of light delivered to one (adapting) slit was adjusted until it produced a criterion fractional reduction in the response to the other (test) slit. The reciprocal of this intensity of the adapting slit was taken as a measure of the effectiveness of the slit in causing light adaptation or inactivation. The effectiveness of the slit in causing light adaptation in normal Musca ommatidia fell as the adapting and test slits were moved farther apart along the ommatidial axis, declining to half its maximal value at a distance of $13 \pm 2$ $\mu \mathrm{m}$. Similar measurements of the effectiveness of a slit in causing light-induced inactivation in the nss mutant of Lucilia also demonstrated localization, declining to half its maximal value at a distance between the slits of $9 \pm 1 \mu \mathrm{m}$. Neither light adaptation nor inactivation by the nss mutation, therefore, appear to be mediated by voltage or by a highly diffusible agent. The results are consistent with the idea that inactivation by the nss mutation replaces adaptation in the mutant photoreceptors.
\end{abstract}

\footnotetext{
Received Apr. 30, 1990; revised Sept. 27, 1990; accepted Oct. 19, 1990.

We thank Dr. K.-W. Yau for his hospitality, use of his laboratory equipment and general support. We also thank Dr. R. S. Stephenson for demonstrating the dissection methods to us and Drs. K.-W. Yau, R. C. Hardie, D. G. Stavenga, and Susan Wood for critical comments on the manuscript. This research was supported by Fight for Sight Inc. to B.M. and by NIH Grants EY06837 to K.-W. Yau EY03529 to B.M., and EY07743 to R.P. K.P. is an Alfred P. Sloan Foundation Fellow.

Correspondence should be addressed to Dr. Baruch Minke, Department of Physiology, The Hebrew University, Hadassah Medical School, P.O. Box 1172, Jerusalem 91010 , Israel.

${ }^{a}$ Present address: Department of Zoology, University of Maryland, College Park, MD 20742.
}

Copyright (C) 1991 Society for Neuroscience $0270-6474 / 91 / 110900-10 \$ 03.00 / 0$
We describe simple preparations of the retinas of the housefly, Musca domestica, and the sheep blowfly, Lucilia cuprina, that allow recording from individual ommatidia in vitro, using a suction pipette similar to that used to record from rod photoreceptors of the vertebrate retina (Baylor et al., 1979; for a preliminary use of this method, see also Becker et al., 1987). The suction-electrode method has 2 advantages when applied to fly photoreceptors. First, the method might prove to be more convenient than the use of intracellular microelectrodes when recording from small photoreceptors, such as those of Drosophila. Recordings from Drosophila are needed to perform functional tests on mutant flies. Second, the suction-electrode method allows the combination of optical and electrophysical measurements on the same isolatcd group of cclls. We therefore used this method to investigate the spatial spread within the photoreceptor of 2 forms of desensitization by bright light. The first is the natural process of light adaptation in ommatidia of white-eyed Musca. The second form of desensitization is that produced by prolonged bright illumination of ommatidia of the no-steady-state (nss) mutant of Lucilia (Howard, 1982, 1984).

Light adaptation reduces the amplitude of the depolarizing events ("bumps") produced by each photon (Dodge et al., 1968; Wu and Pak, 1978). Light adaptation is thought to be the result of a light-induced elevation of cytosolic free calcium ion concentration, $\mathrm{Ca}_{i}$ (Lisman and Brown, 1972). Several studies have demonstrated that adaptation of invertebrate photoreceptors is localized to the area illuminated (Hagins et al., 1962; Hamdorf, 1970; Fein and Lisman, 1975; Payne and Fein, 1983; Baumann and Walz, 1989). This localization probably results from the limited ability of calcium to freely diffuse through cytosol (Fcin and Lisman, 1975). However, the expected small degree of spread due to the diffusion of calcium has not been definitely demonstrated, because this requires accurate measurement of light scatter. The recording methods adopted in the present paper allow measurement of the finely resolved stimulus. We therefore decided to reinvestigate the spatial spread of adaptation, seeking evidence for spread beyond the stimulus.

We also investigate the spatial spread of desensitization in the nss mutant of Lucilia. This desensitization results from a reduced probability that an absorbed photon will produce a bump (Barash et al., 1988), and we therefore call it "inactivation." The mutant photoreceptors do not exhibit light adaptation (Barash et al., 1988), nor is there an increase of $\mathrm{Ca}_{i}$ during illumination of the nss mutant, as evidenced indirectly by the lack of screening pigment migration (Howard, 1984) indicative of an increased $\mathrm{Ca}_{i}$ level (Kirschfeld and Vogt, 1980). Light adaptation and nss mutation therefore appear to act at different 
points in the transduction process, and we wished to determine whether this difference was reflected in their spatial spread. The phenotype of the nss mutant of Lucilia is very similar to that of the transient-receptor-potential (trp) mutant of Drosophila (Cosens and Manning, 1969; Minke et al., 1975; Minke, 1982; Montell et al., 1985; for review, see Pak, 1979). The trp mutant lacks a protein whose sequence has been determined, but whose function in sustaining phototransduction in bright light is unknown (Montell and Rubin, 1989; Wong et al., 1989).

We find that normal light adaptation does spread somewhat beyond the region of scattered light, implying the existence of a messenger of adaptation, such as calcium. However, we can find no evidence for spatial spread of the inactivation due to the nss mutation.

\section{Materials and Methods}

The preparation. White-eyed Musca domestica and the no-steady-state (nss) mutant of the sheep blowfly Lucilia cuprina were used for the experiments. Flies a few hours old were mainly used because they have more flexible ommatidia and thus are more easily drawn into the suction pipette without damage. We used normal Musca flies instead of Lucilia because the former is easier to use in experiments. No differences were found in the light responses of the 2 species. Fly stocks were obtained from Dr. G. G. Foster, CSIRO, Canberra, Australia.

The fly head was dissected from the thorax, hemisected between the eyes, and placed for 10 min in Ringer's solution containing (in $\mathrm{mm}$ ) $\mathrm{NaCl}, 140 ; \mathrm{KCl}, 2 ; \mathrm{MgCl}_{2}, 5$; sucrose, $180 ; \operatorname{HEPES}, 10(\mathrm{pH}, 7)$; and 10 $\mathrm{mg} / \mathrm{ml}$ trypsin. The high sucrose was used to destroy the pigment cells. The hemisected head was then transferred to Ringer's solution without trypsin or sucrose but to which $2 \mathrm{mM} \mathrm{CaCl}_{2}$ was added. The cornea, brain, and part of the lamina were removed from each eye using fine forceps. The partially isolated retina was then transferred to a recording chamber containing oxygenated Ringer's solution (see Baylor et al., 1979), placed on the stage of a Zeiss IM inverted microscope, and viewed with $16 \times(\mathrm{NA}=0.3)$ or $40 \times(\mathrm{NA}=0.6)$ objectives. The dissection was performed according to a method developed by R. S. Stephenson.

Recording and stimulation. Figure 1 shows a single ommatidium of Musca sucked up into a recording pipette. The pipette was fabricated according to the method described in detail by Baylor et al. (1979) and Lamb et al. (1981). Filled with Ringer's solution, the resistance of the pipette was approximately $1 \mathrm{M} \Omega$ when empty and approximately $2 \mathrm{M} \Omega$ with an ommatidium in place. A $120-\mu \mathrm{m}$ length of the ommatidium was typically sucked into the pipette. The axons of the photoreceptors remained intact during the recordings.

The light stimuli were created by passing 2 green $(530 \mathrm{~nm} ; 10-\mathrm{nm}$ bandwidth) or orange ( $580 \mathrm{~nm} ; 10-\mathrm{nm}$ bandwidth) light beams through a pair of slits mounted on an optical bench (Baylor et al., 1979; Lamb et al., 1981). One slit was used for locally light adapting the cells; the other, for testing sensitivity. The unattenuated intensity of the $530-\mathrm{nm}$ or 580-nm adapting light beam at the specimen plane, without a slit in place, was $0.05 \mathrm{~mW} / \mathrm{cm}^{2}$ and $0.08 \mathrm{~mW} / \mathrm{cm}^{2}$, respectively; that of the $530-\mathrm{nm}$ test beam was $0.03 \mathrm{~mW} / \mathrm{cm}^{2}$. The images of the slits were focused onto the ommatidium (Fig. 1) using a Zeiss condenser objective (Epiplan $8 \times ; \mathrm{NA}=0.2$ ). The widths and relative positions of the slits were controlled by micrometers.

The major class of fly photoreceptors have a blue-green rhodopsin and orange metarhodopsin with peak absorption at $490 \mathrm{~nm}$ and 570 $\mathrm{nm}$, respectively. In order to preferentially stimulate these cells and not the minor class of photoreceptors, absorbing mainly at the short-wavelength range, we used long-wavelength stimulation. Such a stimulation also prevented the induction of afterpotentials, which would distort our measurements (Minke, 1986). We mainly used 530-nm light in order to obtain sufficient effective intensity required for our adapting lights.

The intensity profiles of the light slits were measured by placing an $86-\mu \mathrm{m}$-diameter pinhole in the focal plane of the microscope objective and sampling the light emerging through this hole with a photomultiplier tube (Gamma Scientific Inc. Model 2020). To determine the intensity profile of a light slit, the center of the ommatidium was focused onto the pinhole, and the light slit was moved in steps of $1 \mu \mathrm{m}$ along the ommatidium. The light intensity reaching the photomultiplier was plotted as a function of the distance of the center of the light slit from the pinhole, expressed as a percentage of its maximum at the nominal center

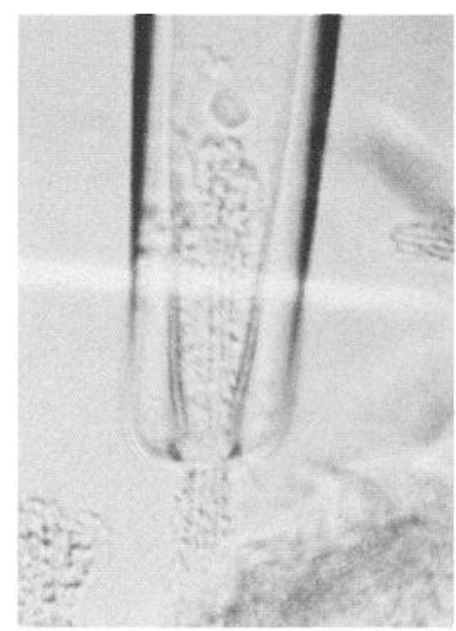

Figure 1. A single ommatidium of Musca within a suction pipette. The plate illustrates a typical configuration of the recording and stimulation procedure photographed from the inverted microscope $(40 \times$; $\mathrm{NA}=0.6$, objective). A slit of light of $5 \mu \mathrm{m}$ diameter can be observed at the middle of the ommatidial portion that was drawn into the pipette.

of the slit. Very little variation in the slit profiles was observed between measurements on several ommatidia (e.g., cf. Figs. 6, 9). Convolution integrals of the intensity profiles of the slits were calculated using ASYST software (Asyst Software Technologies Inc.). The pipette current was measured with a standard current-to-voltage converter (List EPC7).

Derivation of the convolution integral of the light intensity profiles of the adapting and test light slits. We determined the effectiveness of a slit of light in causing light adaptation or inactivation by increasing the intensity of the adapting slit until a criterion reduction in the response to a nearby test slit was attained (see Results). We took the reciprocal of the intensity of the criterion adapting light, $1 / I_{c}$, as a measure of the effectiveness of light adaptation or inactivation.

For the nss mutant, the spatial profile of $1 / I_{c}$ followed closely the convolution integral of the intensity profiles of the inactivating and test light slits (see Results, Fig. 9). A simple derivation of this relationship follows, based on the premise that a photon delivered from a test flash is ineffective if it arrives within a very short distance, $\Delta x$, of a site in the rhabdomere previously hit by a photon delivered by an inactivating flash.

The intensity profiles of the inactivating and test slits along the length, $x$, of the rhabdomere, $I_{1}(x)$ and $I_{2}(x)$, respectively, can be calculated from the product of the peak intensities of the slits $I_{a}$ and $I_{t}$, respectively, and their normalized intensity profiles, $P_{1}$ and $P_{2}$. If the distance between the nominal centers of the slits is $d$, then

$$
I_{1}(x)=I_{a} \cdot P_{1}(x),
$$

and

$$
I_{2}(x)=I_{t} \cdot P_{2}(x-d) .
$$

At a point $x$ along the rhabdomere, the density, $N_{i}(x)$, of ineffective test photons is given by

$$
N_{i}(x)=D_{i}(x) \cdot N(x),
$$

where $D(x)$ is the density of inactive sites, and $N(x)$ is the number of test photons incident within $\Delta x$ of $x . D_{i}(x)$ and $N(x)$ are determined by the intensity profiles of the slits:

$$
D_{i}(x)=k \cdot I_{1}(x),
$$

and

$$
N(x)=\Delta x \cdot I_{2}(x),
$$

where $k$ is the quantum efficiency of the creation of inactive sites.

If the rhabdomere extends from $x=\chi_{1}$ to $x=\chi_{2}$, then the total number of ineffective test photons incident on the rhabdomere, $N_{i}$, is given by the integral of $N_{i}(x)$ from $\chi_{1}$ to $\dot{\chi}_{2}$, which by Equation 3 , is the sum of the products obtained when the 2 overlapping distributions, $D_{i}(x)$ and $N(x)$, are multiplied together point by point. Substituting Equations 4 and 5 into 3 and integrating, we have 


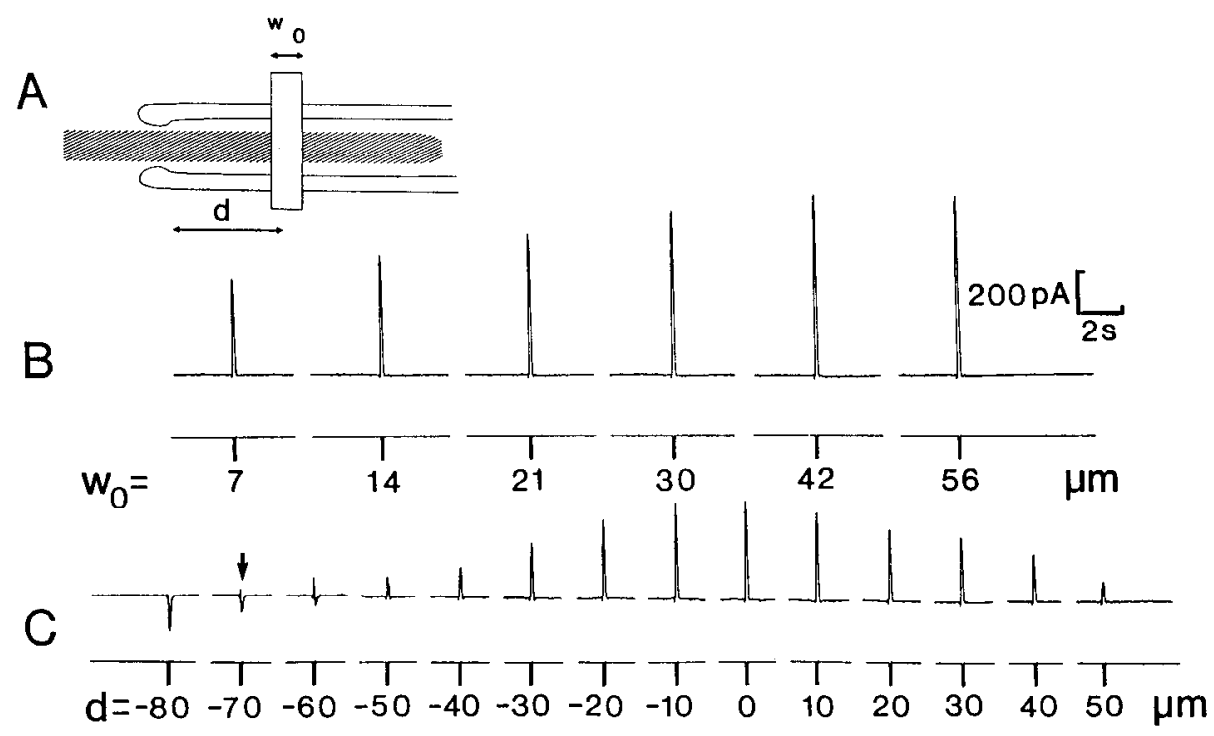

Figure 2. Spatial sensitivity to light of a single ommatidium of Musca measured with a moving stimulus. A, A diagram (not to scale) showing the ommatidium within the recording pipette. $B$, Measurements of light-induced currents (LIC) in response to increasing width, $w$, of the stimulating light slit (as indicated). The stimulating slit was positioned at the location " 0 " (see $C$ ) and expanded symmetrically. $C$, Measurements of LIC in response to $7-\mu \mathrm{m}$ slit at various locations along the ommatidium. Positions -70 and 50 indicate the opening edge of the pipette and the top of the ommatidium, respectively (see $A)$. When the stimulating slit was located outside the pipette $(d=-80 \mu \mathrm{m})$, the LIC had a reverse polarity, indicating that the scction of the ommatidium within the pipette can act as both a current source and a sink. The relative intensity of the orange (580-nm interference filter) stimulus $(I)$ was $-\log I=1.2$. All responses are from a single ommatidium. In each current trace, an upward deflection indicates the flow of current out of the recording pipette. The position of the end of the pipette was at $-70 \mu \mathrm{m}(C$, arrow). Usually, the current reversed $5-10 \mu \mathrm{m}$ inside the pipette.

$$
N_{i}=k \cdot \Delta x \int_{x_{1}}^{x_{2}} I_{1}(x) \cdot I_{2}(x) d x .
$$

Substituting for $I_{1}(x)$ and $I_{2}(x)$, using Equations 1 and 2, we have the following convolution integral, which describes the total number of ineffective test photons as a function of the separation, $d$, between the centers of the slits:

$$
N_{i}(d)=k \cdot \Delta x \cdot I_{a} \cdot I_{t} \int_{x_{1}}^{x_{2}} P_{1}(x) \cdot P_{2}(x-d) d x .
$$

In our experiments, we determined the intensity, $I_{c}$, of the adapting slit that caused a criterion degree of inactivation of the response to the test slit. A criterion number, $N_{c}$, of test photons would then be ineffective at each slit separation. The variation of $I_{c}$ with separation, $d$, can be described by a modified form of Equation 7:

$$
\frac{1}{I_{c}(d)}=k \cdot \Delta x \cdot \frac{I_{t}}{N_{c}} \int_{x_{1}}^{x_{2}} P_{1}(x) \cdot P_{2}(x-d) d x .
$$

The reciprocal of $I_{c}$ should vary with slit separation in proportion to the convolution integral of the slit intensity profiles, as observed for the data from the nss mutant (see Results).

\section{Results}

At the beginning of every experiment, the sensitivity of the recorded ommatidium to flashes of light was determined by positioning a light slit at different positions along its length (Fig. $2 A, C$ ). When the slit was positioned over the portion of the ommatidium outside the pipette, flashes evoked a transient flow of current into the pipette (negative deffections in Fig. 2C). When the slit was positioned over the portion of the ommatidium within the pipette, the direction of current flow upon illumination reversed. The section of the ommatidium within the pipette therefore functioned as a sink of current when illuminated within the pipette and as a source of current when illu- minated outside. Movement of the slit along the portion of the ommatidium within the pipette typically revealed a $30-40-\mu \mathrm{m}$ region of the ommatidium where sensitivity to light was highest (Fig. 2C). Recordings from Musca or Lucilia ommatidia were essentially identical in their spatial profile of sensitivity.

With the stimulus centered on the region of highest sensitivity, the amplitude of the response increased monotonically as the slit width was expanded to cover the entire region of high sensitivity (Fig. $2 B$ ).

The direction of current flow recorded during illumination is consistent with many other studies that have shown that the illumination of invertebrate microvillar photoreceptors results in a flow of inward current that is localized to the area illuminated (Hagins et al., 1962; Millecchia and Mauro, 1969; Fein and Payne, 1989). Therefore, during localized illumination of the fly ommatidium within the suction pipette (Fig. 3, slit), current (indicated by arrows in Fig. 3) presumably enters the photoreceptors (phr) through light-dependent ion channels in the illuminated region (Fig. 3, shaded area). Part of this current (k) returns by passing out of the photoreceptors through leakage channels located in the portions of the photorcceptors' plasma membranes that lie within the pipette (p). The rest of the current (j) leaves the photoreceptors through leakage and voltage-activated channels located in the plasma membranes outside the pipette. We measured the fraction of the latter current that does not leak back into the pipette through the imperfect seal between the photoreceptors and the pipette (Fig. 3) or through the cavity at the center of the ommatidium (not shown in Fig. 3). The electrical resistances of the leakage channels, the pipette seal, the central cavity, and the shaft of the pipette determine the fraction of the light-sensitive inward current that we record.

During localized illumination of the ommatidium outside the pipette or very close to the pipette tip, the flow of current re- 


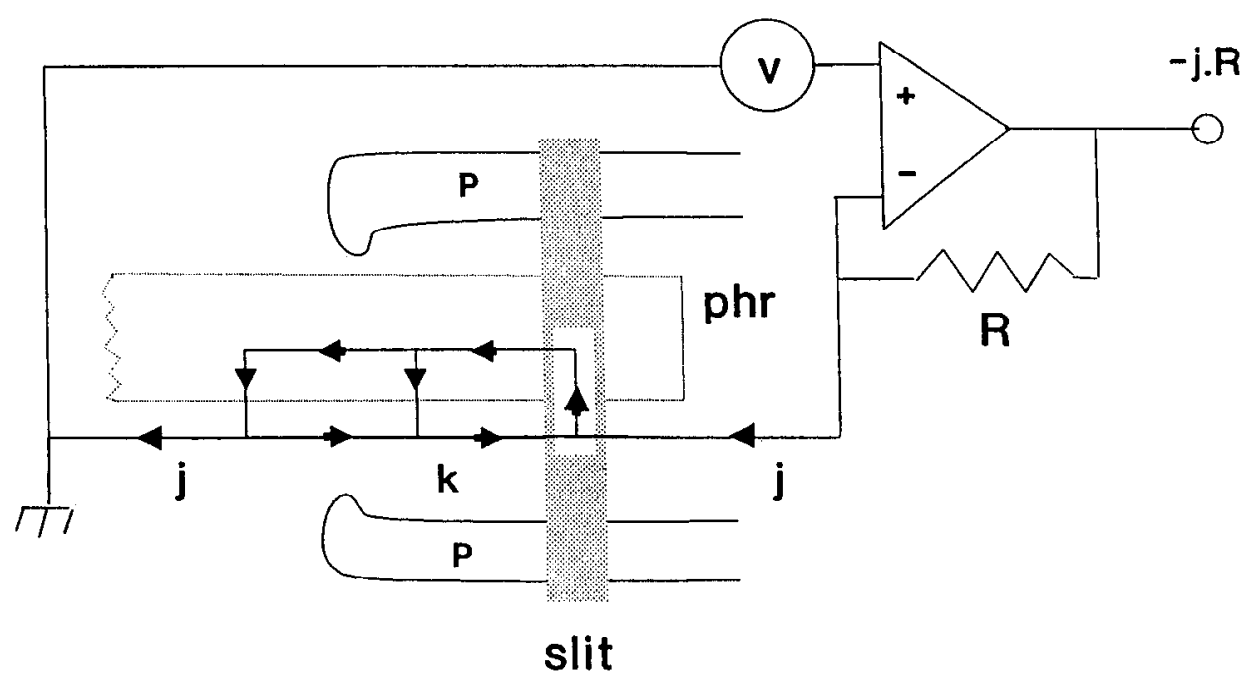

Figure 3. Diagram showing the flow of current (indicated by arrows) during suction-electrode recording from a fly ommatidium. For simplicity, only a single photoreceptor ( $p h r$ ) of the ommatidium is shown within the pipette (p). Of the current that enters the illuminated region of the photoreceptor, the current-to-voltage converter measures only the fraction, $j$, that exits the photoreceptor outside the pipette and does not leak back into the pipette. The remaining fraction of the photocurrent, $k$, is undetected. The resistance, $R$, of the current-to-voltage converter determines the converter's output. Electrode and junction potentials are offset by the voltage source $V$. versed (see Figs. 2, 4). This reversal typically began 5-10 $\mu \mathrm{m}$ inside the pipette. In this case, we recorded the fraction of the light-sensitive current that exits, the photoreceptors within the pipette, and the light-sensitive current that does not return through the pipette seal.

\section{Intensity-response relationship of Musca ommatidia}

The $7-\mu \mathrm{m}$ slit of light used in Figure 2 was centered on the most sensitive region of the ommatidium within the pipette, and 1-sec-duration flashes of increasing intensity were delivered (Fig. 4). Dim illumination produced a constant current accompanied by an increased noise level that indicated the random summation of many single photon events (Kirschfeld, 1966). The amplitude of the initial response rose with increasing stimulus intensity, but the response rapidly declined to a sustained plateau during illumination at the higher intensities due to light adaptation (Laughlin and Hardie, 1978). At the highest intensities, a negative-going phase (notch) was often observed after the initial transient but before the plateau. This notch was more pronounced in our recording method than in the usual intracellular recording. All of these features of the current recorded during illumination (except for the negative phase of the notch) are also observed in records of the membrane potential recorded from $M$ usca photoreceptors in situ using intracellular microelectrodes (Scholes, 1973).

\section{Spatial profile of adaptation in Musca ommatidia}

We wished to determine whether light adaptation due to a slit of light spread significantly within the photoreceptor, further than could be accounted for by light scatter. To quantify our data, we chose to measure the effectiveness of an adapting slit of light in desensitizing the response to a test slit placed at different distances from the adapting slit. Figures 2, 5, and (later) 8 show that sensitivity to light varies along the ommatidium. In order to avoid corrections for these variable sensitivities, we used a stationary test light and moved the adapting light.

We projected $25-\mu \mathrm{m}$ slits of light onto the most sensitive region of the ommatidium. One stationary slit was placed at the center of the region of highest sensitivity. The sensitivity of the ommatidium at the stationary test point was determined by delivering $100-\mathrm{msec}$ light flashes. The other, adapting slit could be moved on either side of the test slit, and its intensity could be adjusted. For a series of distances, $d$, between the centers of the slits, we determined the intensity, $I_{c}$, of a 5-sec flash delivered to the adapting slit that was required to adapt the response to the test slit by a criterion fractional amount, usually between $40 \%$ and $60 \%$ of the dark-adapted amplitude. Figure 5 illustrates responses to the $5-\mu \mathrm{m}$ slits when the adapting and test slits were superimposed (Fig. $5 A$ ) and when they were placed $10 \mu \mathrm{m}$ apart

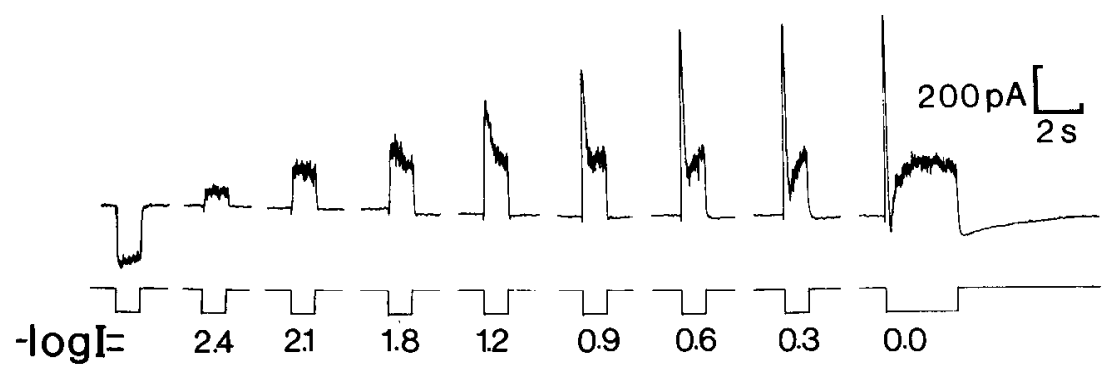

Figure 4. Intensity-response function of a single ommatidium of Musca. The upper row shows LICs in response to increasing intensities of orange light (580-nm interference filter) with relative intensity as indicated below the bottom traces of the light monitor. The first response (negative deflection) was recorded when the $7-\mu \mathrm{m}$ stimulating slit was located $10 \mu \mathrm{m}$ outside the pipette with relative intensity of $-\log I=1.2$. The other responses were recorded when the stimulating slit was positioned within the pipette $70 \mu \mathrm{m}$ above the opening edge of the pipette (see Fig. 2). The small negative deflection before most of the responses is an artifact created by electrical coupling between 1 shutter controller and the EPC7 amplifier. 

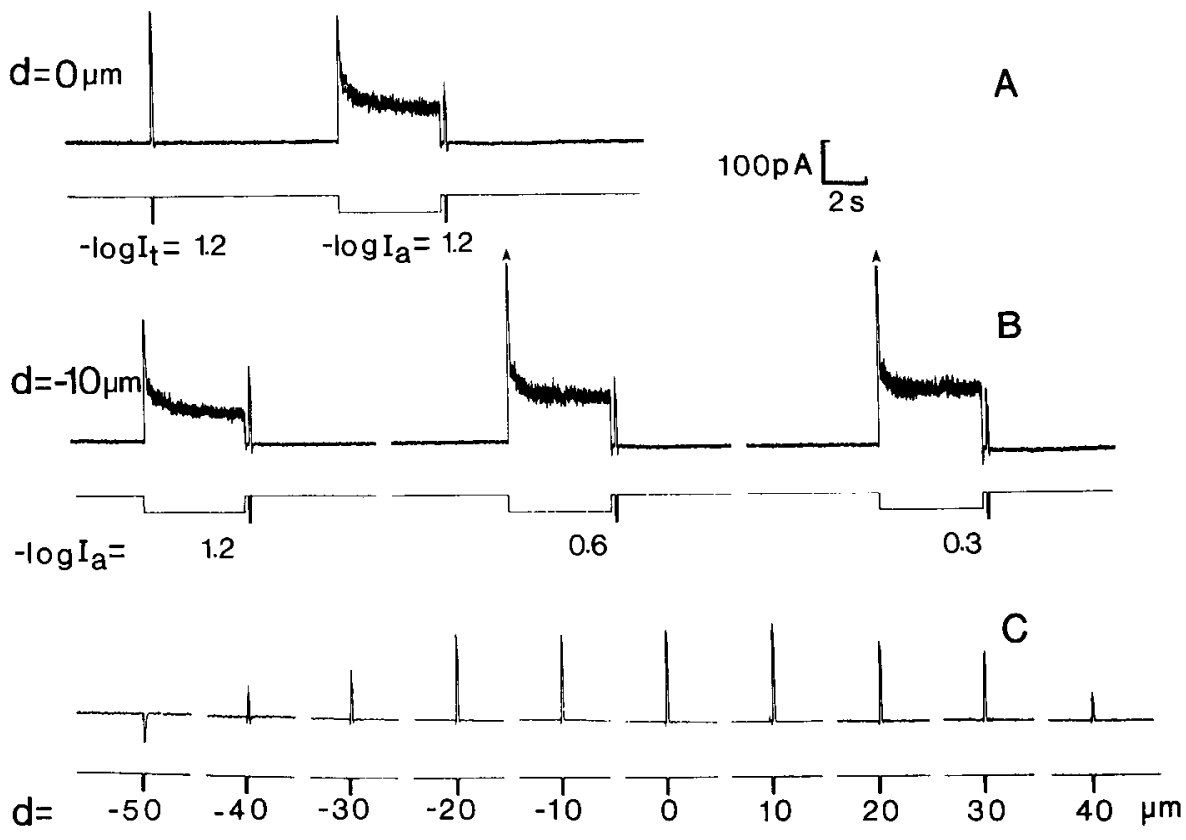

Figure 5. Spread of light adaptation as a function of distance from the location of 5- $\mu \mathrm{m}$ green adapting light slit in a single ommatidium of $M$ usca. The adapting and test light slits consisted of $5-\mu \mathrm{m}$ slits $(530-\mathrm{nm}$ interference filter) that were initially both superimposed by eye at location $0(d=$ $0 \mu \mathrm{m}$; Figs. $2 C, 5 C) . A$, The dark-adapted ( $2 \mathrm{~min})$ response to 0.1 -sec stationary test light $(-\log I=1.2)$ followed by responses to the 5 -sec adapting light ( $-\log I_{a}=1.2$ ) followed (after 0.2-sec dark interval) by the test light. $B$, A repetition of the paradigm of $A$ when the adapting light was moved to position $-10 \mu \mathrm{m}$ (see $C$ ). There was a clear increase in the response to the constant test light (decrease in light adaptation) when the adapting light of $-\log I=1.2$ intensity was located $10 \mu \mathrm{m}$ away from the test light. To measure the effectiveness of the adapting light at a distance of 10 $\mu \mathrm{m}$, the intensity of the adapting light was increased to $-\log I=0.6$ (middle trace) and then to $-\log I=0.3$ (right trace). The amplitude of the response following the $-\log I=0.3$ adapting light is similar to the amplitude of the test light in the control $(d=0 \mu$ m), indicating that the effectiveness of the adapting light declined roughly by $0.9 \log$ units at a distance of $10 \mu \mathrm{m}$. Arrowheads indicate that the peak transient of the response is off scale. $C$, The spatial sensitivity of the ommatidium studied in $A$ and $B$. The moving 5- $\mu \mathrm{m}$ light slit came from the adapting light beam with relative intensity of $-\log I=1.5$ All responses are from a single ommatidium of $M u s c a$ fly.

(Fig. 5B). Adaptation of the response to the test slit is more effective when the centers of the slits are superimposed. In order to produce the criterion amount of adaptation when the centers of the slits were $10 \mu \mathrm{m}$ apart, the intensity of the adapting slit, $I_{c}$, had to be increased by $0.9 \log$ units. At each slit separation, an accurate value of $I_{c}$ was obtained by interpolation as follows: The amplitude of the response to $I_{t}$ was plotted as a function of $-\log I_{a}$ for 3-5 values of $I_{a}$. $I_{c}$ was determined by finding the valuc of $-\log I_{a}$, for which the interpolated response amplitude matched the criterion.

As the distance, $d$, separating the centers of the slits increased, so did $I_{c}$. The reciprocal of $I_{c}$ at various distances, $d,\left[I_{c}(d)\right]$, relative to its value at $d=0,\left[I_{c}(0)\right]$ was taken as a measure of the effectiveness of the adapting slit. Figure $6(0)$ illustrates the decline of the effectiveness of the adapting slit, defined in this way, as $d$ increases on either side of the test slit. The half-width of the effectiveness of the adapting slit was $13 \mu \mathrm{m}$ for this ommatidium. For a total of $5 \mathrm{ommatidia}$ similarly investigated, the mean half-width was $13.2 \pm 1.9 \mu \mathrm{m}( \pm \mathrm{SE})$. The inset in Figure 6 plots the decline of $I_{c}(d)$ on semilog coordinates for positive ( $\square$ ) and negative ( $(\theta)$ values of $d$. For values of $d$ greater than $5 \mu \mathrm{m}, \log \left(1 / I_{c}\right)$ declined approximately linearly as $d$ increased, displaying a space constant of $6.7 \mu \mathrm{m}$ for an $e$-fold decline. A total of 5 ommatidia exhibited a mean space constant of $7.6 \pm 1.4 \mu \mathrm{m}( \pm \mathrm{SE})$.

In each experiment for the same ommatidium, we measured the normalized intensity profile of the adapting slit by scanning the ommatidium with a photometer (see Materials and Meth- ods). The broken line in Figure 6 illustrates this profile. The solid line illustrates the convolution integral (Eq. 8; see Materials and Methods) of the normalized intensity profiles of the test and the adapting slits, which indicates the probability density distribution for the coincidence of photons delivered by the test and adapting slits. Both the normalized intensity profiles of the adapting slit and the convolution integral are narrower than the curve describing the reciprocal of $I_{c}$.

\section{Experiments performed on the nss mutant of Lucilia \\ Characteristics of the response to light}

Ommatidia from nss Lucilia were prepared and drawn into micropipettes in an identical manner to those of Musca. The spatial sensitivity profile and the magnitude of responses to brief flashes of light were similar to those of $\mathrm{Musca}$ ommatidia (see Figs. $5 C, 8 C$ ). However, the responses of the nss ommatidia declined during prolonged bright flashes, due to the mutationinduced inactivation, with no plateau phase (Fig. 7). The lack of any appreciable sustained response to a bright $50-\mu \mathrm{m}$-wide slit of light is also characteristic of intracellular recordings from this mutant (Howard 1982, 1984; Barash et al., 1988). The decline in the response occurred during illumination by both narrow $(5-\mu \mathrm{m})$ and broad $(50-\mu \mathrm{m})$ slits of light (Fig. 7). The persistence of a small noisy plateau at all but the highest light intensity when illuminated through the $5-\mu \mathrm{m}$ slit is probably due to light scattered at a lower intensity to neighboring parts of the ommatidium. It is clear from Figure 7 that the wide slit has a considerably higher "effective" intensity relative to the narrow 


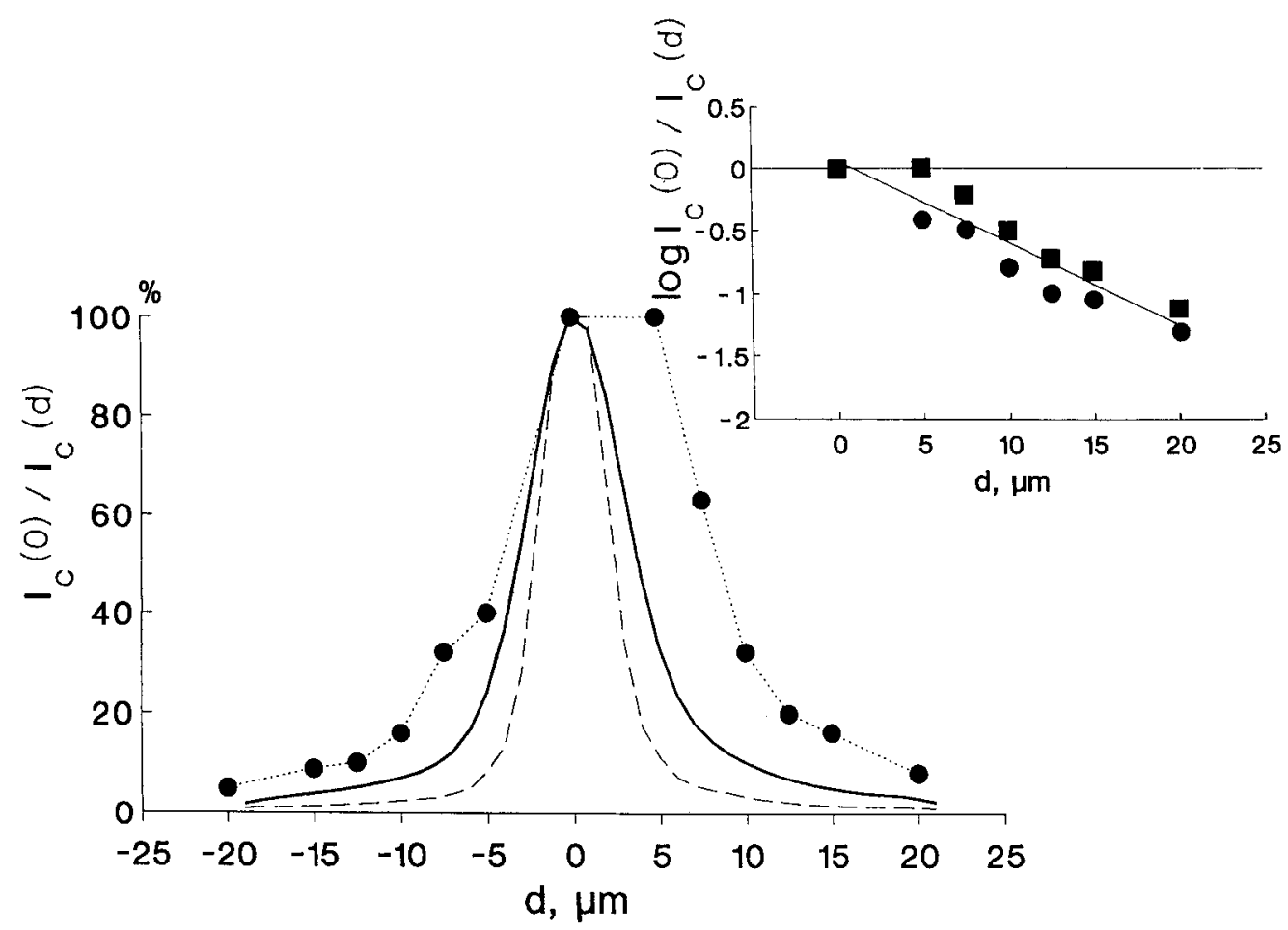

Figure 6. The decline of the effectiveness of the adapting $5-\mu \mathrm{m}$ slit of light as a function of distance, $d$, between the centers of the adapting and test lights. The criterion intensity of the adapting slit, $I_{c}$, was determined for a series of separations, $d$, between the centers of the slits using the method described in Figure 5 and the accompanying text. The resulting distribution of intensities, $I_{c}(d)$, was then normalized relative to the value $I_{c}(0)$ when the slits were superimposed at $d=0$. The reciprocal of the normalized distribution of criterion intensities, $I_{c}(0) / I_{c}(d)$ was taken as a measure of the effectiveness of the adapting slit and is plotted as solid circles. The broken curve is the normalized intensity profile of the adapting slit (see Materials and Methods). The solid curve is the convolution integral of the intensity profiles of the adapting and test slits (Eq. 8). The slight asymmetry of the experimental curve may be due to a small $(\sim 2.5 \mu \mathrm{m})$ misalignment of the centers of the test and adapting slits when the 2 slits were superimposed by eye. Inset. A semilog plot of the experimental data of this figure. The solid circles and squares were plotted for negative and positive values of $d$ (left and right side of the peak in the main figure), respectively. All the data points were measured from the ommatidium of Figure 5.

slit at any given stimulus intensity. The difference between the effective intensities of the wide and narrow slits is probably due to light scatter.

\section{The spatial profle of inactivation of the response of nss ommatidia}

We employed the same methods and paradigm as were used to determine the spatial profile of the effectiveness of light adaptation in Musca ommatidia. Figure 8 illustrates responses when the inactivating and test slits were superimposed (Fig. $8 A$ ) and when they were placed $15 \mu \mathrm{m}$ apart (Fig. $8 B$ ). Inactivation of the response to the test slit is greatest when the slits are superimposed $\left(-\log I_{a}=1.5\right.$; Fig. $8 A$ ). At a separation of $15 \mu \mathrm{m}$ between the adapting and test slits, the adapting light had to be increased to $-\log I_{a}=0.3$ (Fig. $8 B$ ) in order to produce the criterion amount of inactivation; the inactivating slit intensity was therefore increased by $1.2 \mathrm{log}$ units. The desensitization of the nss response following an inactivating light flash is more profound than that caused by normal light adaptation. Therefore, the chosen criterion of desensitization was usually larger than those chosen for the experiments on light adaptation in Musca, between $50 \%$ and $90 \%$ inactivation.

Figure 9 illustrates the effectiveness of the inactivating light (the reciprocal of $I_{c}$ ) as a function of the distance, $d$, between the slits ( ). The half-width of the effectiveness of the inactivating light was $8.3 \mu \mathrm{m}$ for this ommatidium. For a total of 4 ommatidia similarly investigated, the mean half-width was 9.2 $\pm 1.0 \mu \mathrm{m}( \pm \mathrm{SE})$.

The inset in Figure 9 plots the decline of the normalized $I_{c}(d)$ on semilog coordinates for positive $(\square)$ and negative $(0)$ values of $d$. The $\log \left(1 / I_{c}\right)$ declined approximately linearly as $d$ increased, displaying a space constant of $6.9 \mu \mathrm{m}$ for an $e$-fold decline. A total of 4 ommatidia exhibited a mean space constant of $6.6 \pm 1.0 \mu \mathrm{m}( \pm \mathrm{SE})$.

For the same ommatidium, we measured the normalized intensity profile of the inactivating slit by scanning the ommatidium with a photometer (see Materials and Methods). The broken line in Figure 9 illustrates this profile. The solid line illustrates the convolution integral (Eq. 8; see Materials and Methods) of the normalized intensity profiles of the test and the inactivating slits, which indicates the probability density of coincidence of photons delivered by the 2 slits. The curve describing the reciprocal of $I_{c}$ is broader than the normalized intensity profile of the inactivating slit, but it lies close to the convolution integral of the 2 slits.

\section{The time course of recovery from desensitization}

The above experiment indicated that inactivation in the nss mutant is highly localized to the area illuminated. This effect may replace the spread of adaptation that is present in the nonmutant flies (Fig. 6; see Discussion).

We decided to look at the time course of recovery from inactivation for evidence of a diffusible messenger of inactivation. 


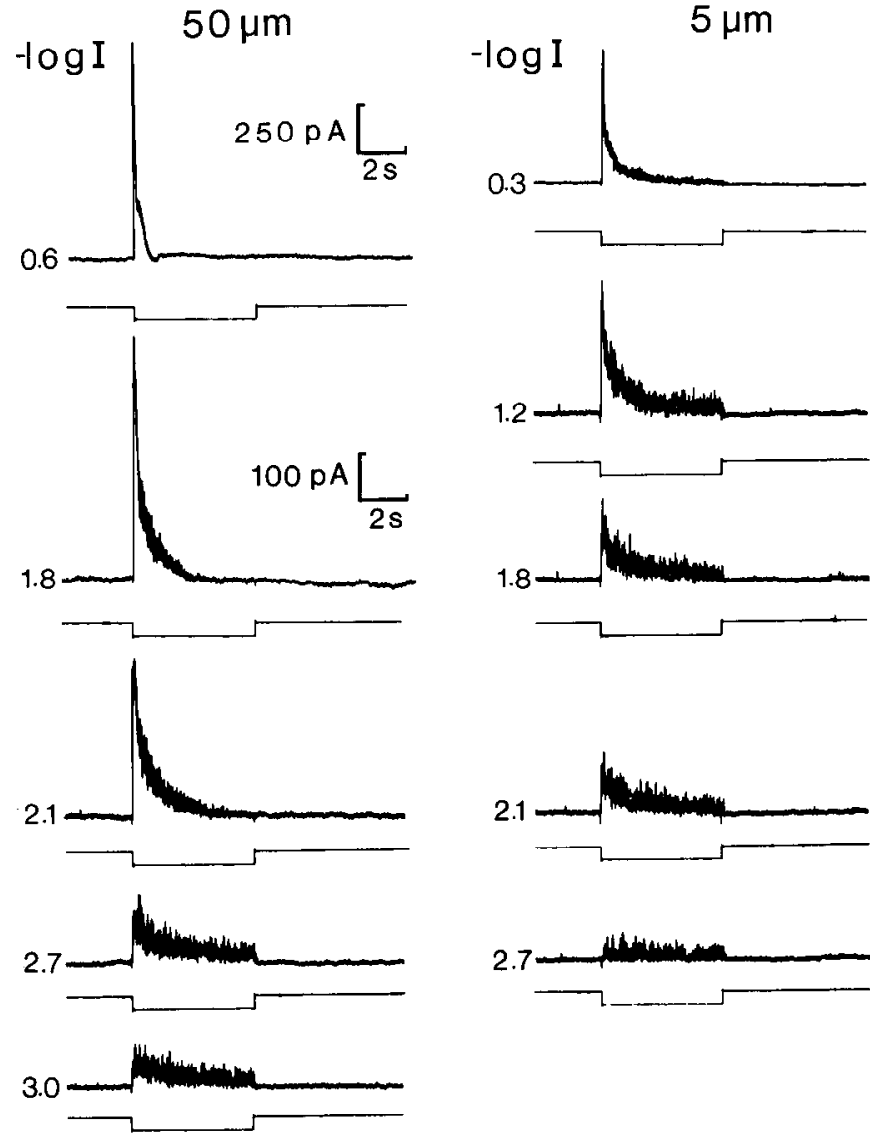

Figure 7. The wave forms of the LICs of the nss mutant of the sheep blowfly Lucilia, in response to increasing intensities of orange light slits of 5- $\mu \mathrm{m}$ and $50-\mu \mathrm{m}$ widths. Note a change in gain for the upper row. All responses were recorded from a single ommatidium.

If such a substance exists, the time taken for recovery of the response under a slit might be due to the time taken for this substance to diffuse from the inactivated region. The time course of recovery would then be expected to be highly dependent on the width of the inactivating slit. Crank (1975) presents a solution to the diffusion equation for the diffusion along a cylinder of a substance from a nonrenewable source of finite width. At the center of the source, the concentration of the substance declines to approximately $50 \%$ of its initial value after a time given by $t=h^{2} / 4 D$, where $h$ is the source width, and $D$ is the diffusion constant of the substance. If we equate this time to that required for $50 \%$ recovery from inactivation, then we would expect the time taken for recovery to increase with the square of the slit width.

We therefore measured the recovery from inactivation of the nss ommatidium following illumination by an adapting narrow light slit of $7 \mu \mathrm{m}$ and wide slit of $48-\mu \mathrm{m}$ width of the same nominal light intensity (Fig. 10). The width of the test slit, upon which the adapting light slit was superimposed, was $5 \mu \mathrm{m}$ to ensure that the area stimulated by the narrow test light was adapted approximately uniformly. A plot of the recovery as a function of time in the dark after adapting light is shown in Figure 11 .

If the time course of recovery were limited by diffusion alone, the time taken for recovery from illumination with the wide adapting slit should increase by a factor of approximately 50 relative to the narrow slit. In fact, we observed only a modest increase in the time for $50 \%$ recovery of the response at the center of the wider slit, from 12 to $18 \mathrm{sec}$ (Fig. 11, $)$ ). This increase could be accounted for by the more profound initial inactivation under the wider slit, possibly due to a much larger light scatter produced by the wider slit resulting in a much higher effective intensity (see Fig. 7). The time for recovery from 10\% to $80 \%$ of final amplitude was even less affected, actually increasing from 19 to $20 \mathrm{sec}$. Thus, it would appear that the time course of recovery from inactivation is not limited by diffusion of an inactivating substance from the illuminated region. Similar arguments would apply to a model in which inactivation was caused by the depletion of a diffusible substance required to sustain phototransduction (Minke, 1982). We could not, therefore, obtain evidence for the involvement of diffusible messengers or substrates in recovery from inactivation.

\section{Discussion}

\section{The interpretation of current flow during suction-electrode recording from isolated ommatidia}

The relationship between the light stimulus and the current that we recorded is complicated by several factors inherent in our recording method. First, our recordings represent the summed activity of more than 1 photoreceptor with different spectral sensitivity within the ommatidium. However, for the wavelength and light intensities used in this work, only 1 class of photoreceptors was excited (i.e., the peripheral photoreceptors R1-R6). Second, the recordings do not simply reflect the activity of the light-dependent conductance in the photoreceptors' plasma membrane. Fly photoreceptors possess voltage-activated potassium channels that allow current to leak out of the portion of the photoreceptor within the pipette during depolarization by light (Weckstrom et al., 1991). Our recordings from fly ommatidia therefore measure the difference between the currents flowing through the voltage- and light-dependent channels in the portion of the plasma membrane that lies within the pipette. In addition, voltage-activated conductances in the axons and nerve terminals of the photoreceptors may also distort the recordings of the photocurrent. Because of these complexities in the relationship between the stimulus and the recorded current, we decided to use a criterion reduction of a fixed test response when determining the effectiveness of the adapting slit.

It is interesting to contrast our recordings from fly ommatidia with those obtained from vertebrate rod photoreceptors using the suction-electrode method. The insensitivity to membrane potential of the dark current of the rod outer segment and the absence of leakage channels ensures that changes in current flowing through the light-sensitive channels are recorded virtually undistorted (Baylor and Lamb, 1982; Baylor and Nunn, 1986). Only the fraction of the current that returns through the pipette seal is undetected.

\section{The nss-induced inactivation is highly localized}

The spatial profile of the light slit in causing inactivation in the nss ommatidium followed closely the convolution integral of the intensity profiles of the adapting and test light slits (Fig. 9). This result indicated that the process affected by the nss mutation is confined to the illuminated area.

Our finding that the time taken for recovery from inactivation is relatively insensitive to slit width also provides no evidence for a diffusible messenger of inactivation in the nss mutant.

We could not find evidence for significant spread of the ef- 


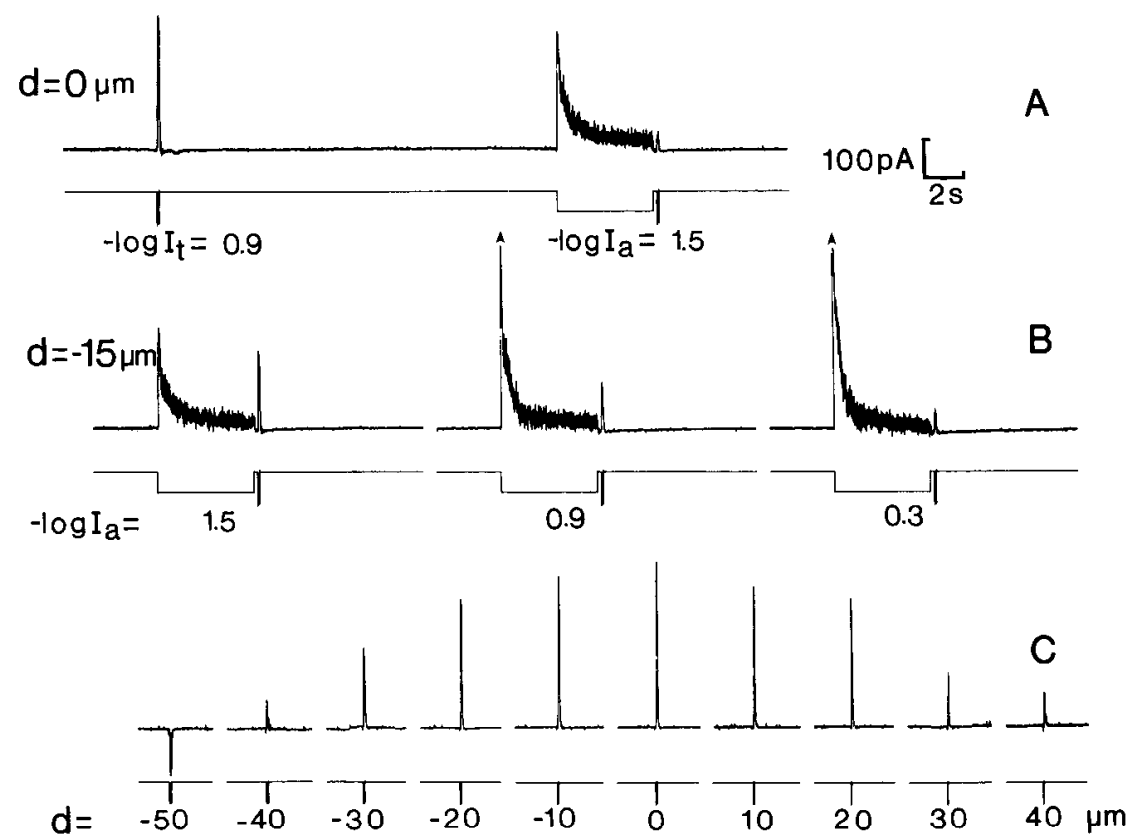

Figure 8. Spread of inactivation as a function of distance from the location of $5-\mu \mathrm{m}$ green adapting light slit in a single ommatidium of the nss mutant. The experimental paradigm was identical to that of Figure $5 . A$, The response of the dark-adapted ommatidium to the test light $\left(I_{i} ; 5-\mu \mathrm{m}\right.$ slit, $530-\mathrm{nm}$ interference filter). It also shows the response to the inactivating slit $\left(I_{a} ; 5-\mu \mathrm{m}\right.$ slit, $530-\mathrm{nm}$ interference filter), which was shortly $(0.2 \mathrm{sec})$ followed by the constant test light. $B$, A repetition of $A$ when the adapting light was moved to position $-15 \mu \mathrm{m}$. A large increase in the response to $I$, was observed when $I$ was unchanged. A suppression of the response to the test light to slightly above the criterion level was obtained when $I_{a}$ was increased to $-\log I_{a}=0.9$ ( middle trace). Increasing the intensity of $I_{a}$ to $-\log I=0.3$ suppressed the response of the test light to the criterion level (right trace). Arrowheads indicate that the peak amplitude of the transient phase is off scale. $C$, The spatial sensitivity of the ommatidium studied in $A$ and $B$. The moving $5-\mu \mathrm{m}$ light slit came from the inactivating light beam with relative intensity of $-\log I=1.2$. fectiveness of inactivation, beyond that expected from light scatter. Nevertheless, a question arises as to why the small spread of adaptation observed in the wild type (Fig. 6) is not observed in the mutant (Fig. 9). If adaptational spread were summed with inactivation in the mutant, then we would expect the spread of the effectiveness of inactivation in the mutant to be even broader than that of adaptation in the normal flies. This is because it would take less light to inhibit the response at a distance to the criterion level. The fact that the spread of inactivation for nss
(Fig. 9) is narrower than the spread of adaptation in the wild type suggests that adaptation is replaced by nss inactivation, rather than summed with it. This conclusion is consistent with the evidence that there is no light adaptation in the nss mutant, and that the concentration of the putative messenger of adaptation, $\mathrm{Ca}$, is unusually low in the nss mutant (see introductory remarks).

The nature of the agent that is responsible for the reduction in the efficiency of phototransduction during prolonged illu-

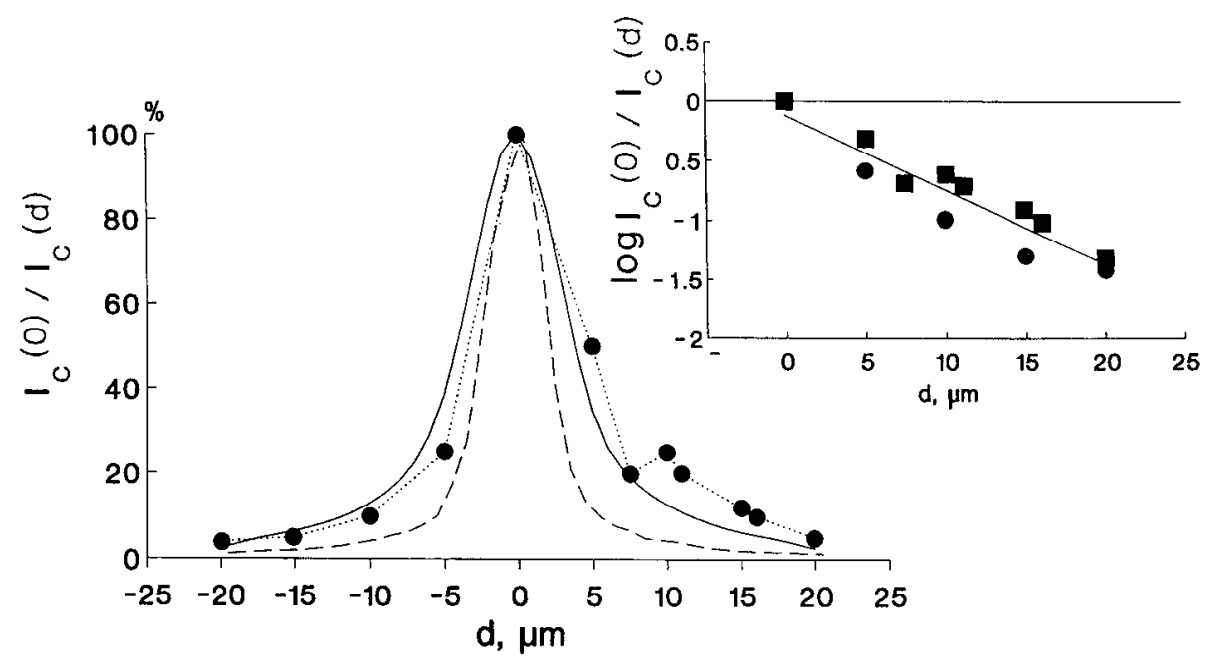

Figure 9. The decline of the effectiveness of the inactivating $5-\mu \mathrm{m}$ slit of light as a function of distance between the inactivating and test slits. The experimental paradigm and presentation are the same as in Figure 6. The solid circles represent the normalized reciprocal of the relative intensity of the inactivating $\left[I_{c}(0) / I_{c}(d)\right]$ slit needed to suppress the response to the constant test slit (which was located at various distances from the moving adapting light) to a criterion level at various distances $(d)$. The criterion level was determined when the inactivating and test lights were superimposed. The broken curve is the normalized intensity profile of the inactivating light. The solid curve is the convolution of the intensity profiles of the adapting and test slits (Eq. 8). Inset. A semilog plot of the experimental data. The solid circles and squares were plotted for negative and positive values of $d$, respectively. All data points were taken from the ommatidium of Figure 8 . 
Figure 10. The recovery of the response of an $n s s$ ommatidium following inactivating slits of 7- $\mu \mathrm{m}$ (left) and 48$\mu \mathrm{m}$ (right) widths. The relative light intensity of the 530-nm inactivating and test slits was $-\log I=1.5$. The width of the test light was $5 \mu \mathrm{m}$ to ensure that the ommatidial area stimulated by the narrow test slit was adapted (roughly) uniformly. The interrupted baseline indicates a 38 -sec pause.

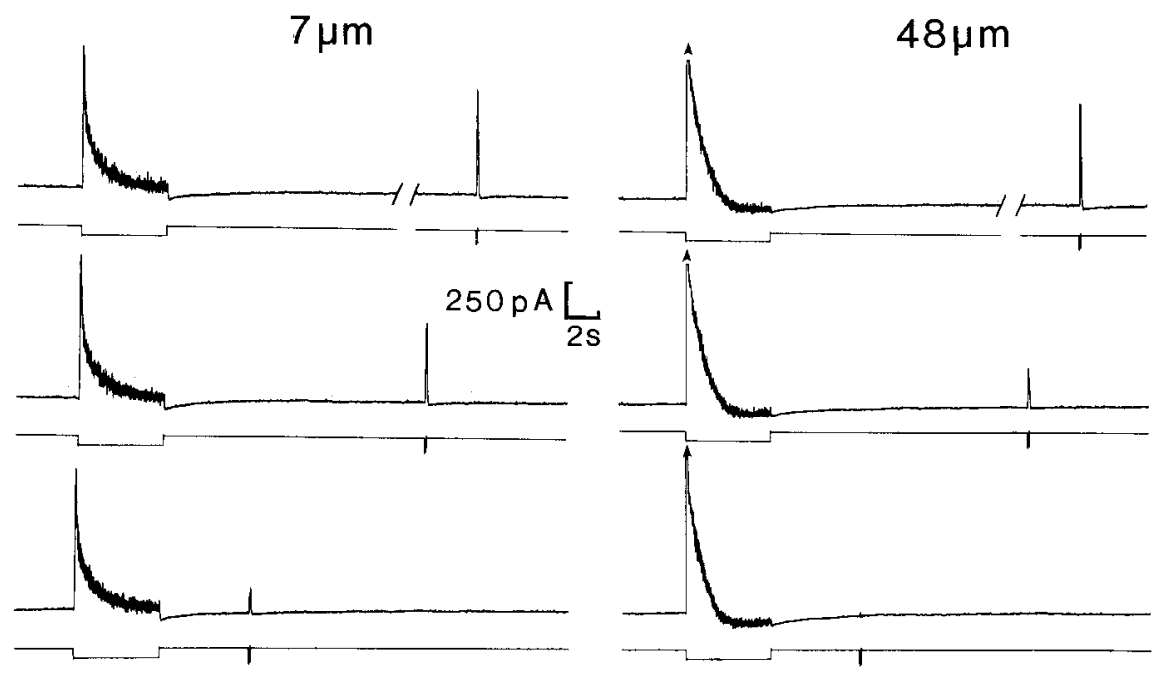

mination of the nss mutation is unknown. Our results rule out mediation of the desensitization by the production or depletion of any agent that readily spreads within the photoreceptor, such as $\mathrm{Na}^{+}$or $\mathrm{K}^{+}$ions. The results also rule out mediation via membrane potential. Our results are consistent with the depletion of an active intermediate that is highly localized within the photoreceptor. The trp mutant of Drosophila, which has a similar phenotype to nss (Barash et al., 1988), lacks a novel integral membrane protein. The scquencc of this protein has been determined (Montell and Rubin, 1989; Wong et al., 1989), but its function is unknown. A pharmacological study by Hochstrate (1989) also suggests that the trp phenotype is caused by the malfunction of a transmembrane protein. Our study further suggests that the deficient process resulting from the malfunctioning protein is also highly localized.

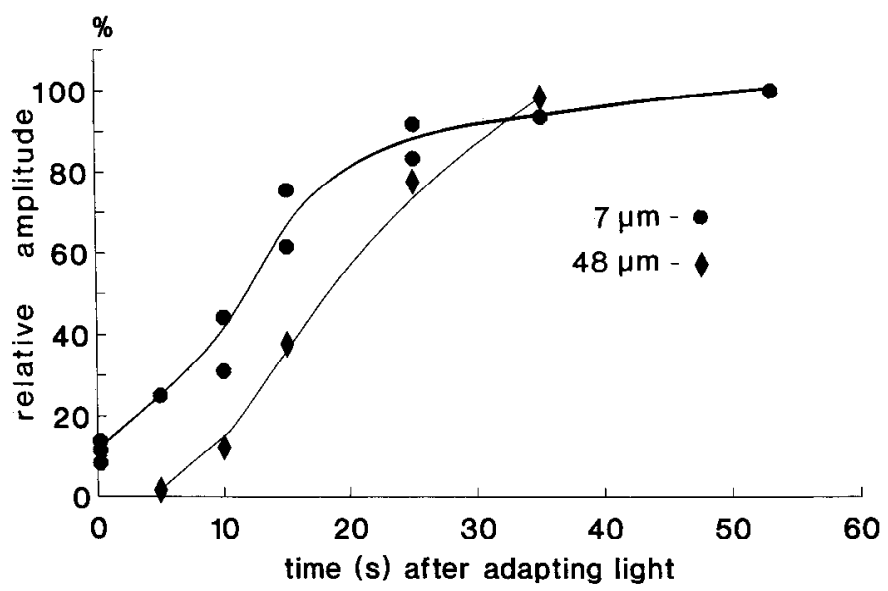

Figure 11. A plot of the recovery of the response of the nss ommatidium of Figure 10 as a function of time in the dark after illumination by inactivating slits of 2 different widths. The amplitude of the response to the 5- $\mu \mathrm{m}$ test slit applied at various times following the superimposed $7-\mu \mathrm{m}$ or $48-\mu \mathrm{m}$ inactivating slit was divided by the amplitude of the response to the test slit in dark-adapted conditions ( $\sim 1$ min dark) and plotted as a function of the time in darkness between the inactivating and test flashes. Each point represents a separate experimental run. The relative light intensity for the $530-\mathrm{nm}$ adapting and test slits was $-\log$ $I=1.5$.

\section{The spatial spread of adaptation in Musca ommatidia}

For normal light adaptation within Musca ommatidia, the spatial profile of $1 / I_{c}$ was somewhat broader than that of the convolution integral (Eq. 8) of the slit profiles. Whether this broadness is due to the limited diffusion of the adapting messenger, probably calcium (Lisman and Brown, 1972; Brown and Blinks, 1974), remains to be determined by further experiments and prcfcrably by the direct measurement of the calcium concentration within the ommatidium. The localization of adaptation confirms earlier work using an excised eye slice preparation (Hamdorf, 1970).

\section{References}

Barash S, Suss E, Stavenga DG, Rubinstein CT, Selinger Z, Minke B (1988) Light reduces the excitation efficiency in the nss mutant of the sheep blowfly Lucilia. J Gen Physiol 92:307-330.

Baumann O, Walz B (1989) Calcium- and inositol polyphosphatesensitivity of the calcium-sequestering endoplasmic reticulum in the photoreceptor cells of the honeybee drone. J Comp Physiol A 165: 627-636.

Baylor DA, Lamb TD (1982) Local effects of bleaching in retinal rods of the toad. J Physiol (Lond) 328:48-71.

Baylor DA, Nunn BJ (1986) Electrical properties of the light-sensitive conductance of salamander rods. J Physiol (Lond) 371:115-145.

Baylor DA, Lamb TD, Yau K-W (1979) The membrane current of single rod outer segments. J Physiol (Lond) 288:589-611.

Becker UW, Minke B, Stieve H, Ohlinger C (1987) Light-activated photoreceptor currents of arthropod ommatidia measured with suction electrode. In: New frontiers in brain research. Proceedings of the 15th Gottingen neurobiology conference (Elsver N, Creutzfeld D, eds), p 135. New York: Thieme.

Brown JE, Blinks JR (1974) Changes in intracellular free calcium during illumination of invertebrate photoreceptors. Detection with aequorin. J Gen Physiol 64:643-665.

Cosens DJ, Manning A (1969) Abnormal electroretinogram from a Drosophila mutant. Nature 224:285-287.

Crank J (1975) The mathematics of diffusion, pp 14-16. Oxford: Clarendon.

Dodge FA Jr, Knight BW, Toyoda J (1968) Voltage noise in Limulus ventral cells. Science 160:88-90.

Fein A, Lisman JE (1975) Localized desensitization of Limulus photoreceptors produced by light or by intracellular calcium ion injection. Science 187:1094-1096.

Fein A, Payne R (1989) Phototransduction in Limulus photoreceptors: roles of calcium and inositol trisphosphate. In: Facets of vision (Stavenga DG, Hardie RC, eds), pp 173-185. New York: Springer. 
Hagins WA, Zonana HV, Adams RG (1962) Local membrane current in the outer segments of squid photorcecptors. Nature 194:844-847.

Hamdorf K (1970) Korrelation zwischen Sehfarbstoffgehalt und Empfindlichkeit bei Photorezeptoren. Verh Dtsch Zool Ges 64:148-157.

Hochstrate P (1989) Lanthanum mimics trp photoreceptor mutant of Drosophila in blowfly Calliphora. J Comp Physiol A 166:179-188.

Howard J (1982) Kinetics and noise of transduction in insect photoreceptors. PhD thesis, Australian National University.

Howard J (1984) Calcium enables photoreceptor pigment migration in a mutant fly. J Exp Biol 113:471-475.

Kirschfeld K (1966) Discrete and graded potentials in the compound eye of the fly Musca. In: The functional organisation of the compound eye (Benhard CB, ed), pp 291-307. Oxford: Pergamon.

Kirschfeld K, Vogt K (1980) Calcium ions and pigment migration in fly photoreceptors. Naturwissenschaften 67:S516.

Laughlin SB, Hardie RC (1978) Common strategies for light adaptation in the peripheral visual systems of fly and dragonfly. J Comp Physiol 128:319-340.

Lamb TD, McNaughton PA, Yau K-W (1981) Spatial spread of activation and background desensitization in toad rod outer segments. J Physiol (Lond) 319:463-491.

Lisman JE, Brown JE (1972) The effects of intracellular iontophoretic injection of calcium and sodium ions on the light-response of Limulus ventral photoreceptors. J Gen Physiol 59:701-719.

Millecchia R, Mauro A (1969) The ventral photoreceptor cells of Limulus. III. A voltage-clamp study. J Gen Physiol 54:331-351.

Minke B (1982) Light-induced reduction in excitation efficiency in the trp mutant of Drosophila. J Gen Physiol 76:361-385.
Minke B (1986) Photopigment-dependent adaptation in invertebrates-implications for vertebrates. In: The molecular mechanism of photoreception (Stieve H, ed), pp 241-265. Berlin: Springer.

Minke B, Wu C-F, Pak WL (1975) Induction of photoreceptor voltage noise in the dark in Drosophila mutant. Nature 258:84-87.

Montell C, Rubin GM (1989) Molecular characterization of Drosophila trp locus: a putative integral membrane protein required for phototransduction. Neuron 2:1313-1323.

Montell C, Jones K, Hafen E, Rubin G (1985) Rescue of the Drosophila phototransduction mutant trp by germline transformation. Science 230:1040-1043.

Pak WL (1979) Study of photoreceptor function using Drosophila mutants. In: Neurogenetics: genetic approaches to the nervous system (Breakfield X, ed), pp 67-99. New York: Elsevier.

Payne R, Fein A (1983) Localized adaptation within the rhabdomeral lobe of Limulus ventral photoreceptors. J Gen Physiol 81:767-769.

Scholes J (1973) The electrical responses of the retinal receptors and the lamina in the visual system of the fly Musca. Kybernetik 6:149162.

Weckström M, Hardie RC, Laughlin SB (1991) The role of voltageactivated potassium channels in blowfly photoreceptors. J Physiol (Lond), in press.

Wong J, Schaefer EL, Roop BC, LaMendola JN, Johnson-Seaton D, Shao D (1989) Proper function of the Drosophila trp gene product during pupal development is important for normal visual transduction in adult. Neuron 3:81-94.

Wu C-F, Pak WL (1978) Light-induced voltage noise in photoreceptors of Drosophila melanogaster. J Gen Physiol 71:249-268. 